archives-ouvertes

\title{
Assessing the carbon footprint of the data transmission on a backbone network
}

Marion Ficher, Françoise Berthoud, Anne-Laure Ligozat, Patrick Sigonneau, Maxime Wisslé, Badis Tebbani

\section{To cite this version:}

Marion Ficher, Françoise Berthoud, Anne-Laure Ligozat, Patrick Sigonneau, Maxime Wisslé, et al.. Assessing the carbon footprint of the data transmission on a backbone network. 24th Conference on Innovation in Clouds, Internet and Networks, Mar 2021, Paris, France. hal-03196527

\section{HAL Id: hal-03196527 \\ https://hal.archives-ouvertes.fr/hal-03196527}

Submitted on 12 Apr 2021

HAL is a multi-disciplinary open access archive for the deposit and dissemination of scientific research documents, whether they are published or not. The documents may come from teaching and research institutions in France or abroad, or from public or private research centers.
L'archive ouverte pluridisciplinaire HAL, est destinée au dépôt et à la diffusion de documents scientifiques de niveau recherche, publiés ou non, émanant des établissements d'enseignement et de recherche français ou étrangers, des laboratoires publics ou privés. 


\section{Assessing the carbon footprint of the data transmission on a backbone network}

\author{
Marion Ficher \\ University of Paris - CRI PARIS \\ marion.ficher@cri-paris.org \\ Patrick Sigonneau \\ GIP RENATER \\ patrick.sigonneau@renater.fr
}

\author{
Françoise Berthoud \\ CNRS Grenoble \\ francoise.berthoud@grenoble.cnrs.fr \\ Maxime Wisslé \\ GIP RENATER \\ maxime.wissle@renater.fr
}

\author{
Anne-Laure Ligozat \\ Université Paris-Saclay, CNRS, ENSIIE, LISN \\ anne-laure.ligozat@lisn.upsaclay.fr \\ Badis Tebbani \\ GIP RENATER \\ badis.tebbani@renater.fr
}

\begin{abstract}
The goal of this paper is to assess the carbon footprint of data transmission on a network via a simplified Life Cycle Assessment (LCA) methodology. We did direct measures of the electricity consumption and the IP traffic o $f \mathbf{m}$ ost of the devices required for the transmission between two sites on the backbone network. Our case study aims to compare two conditions for the data transmission, varying infrastructure density and network activity. The results for the peak day are 1.4 gCO2e/GB for the transmission across France and 2 gCO2e/GB for a more local transmission, and respectively $0.6 \mathrm{gCO} 2 \mathrm{e} / \mathrm{GB}$ and $1.1 \mathrm{gCO2e} / \mathrm{GB}$ for an off-peak day.
\end{abstract}

Index Terms-Life Cycle Assessment, Carbon Footprint, Backbone Network Metrology

\section{INTRODUCTION}

The questions that we wish to answer in this paper are the following: What are the greenhouse gas (GHG) emissions of transmitting $1 \mathrm{~GB}$ from A to B? And how does the network structure and activity influence this impact? We focus here on the impact of the backbone network.

Nowadays, the digital sector has an environmental impact; yet impact of data transmission over a network has received relatively little attention. A first $\mathrm{m}$ otivation o $\mathrm{fthis} \mathrm{w}$ ork is to evaluate the carbon footprint of data transmission on a backbone network. In addition, one of our objectives is to be able to assess the impact of changes in the network, so as to minimize its carbon impact.

In this paper, we adopt a simplified LCA m ethodology to assess the GHG emissions due to the transmission of $1 \mathrm{~GB}$ of data on the RENATER backbone. It takes into account the GHG emissions of all devices from the production phase (material extraction and processing, and distribution) to the use phase.

We use direct measures for the electricity consumption and traffic o $\mathrm{nt}$ he a ctive $\mathrm{d}$ evices. T herefore, $\mathrm{o}$ ur a pproach i s a bottom-up one. The network considered is the RENATER network, which is a French network dedicated to the Education and Research community. One of the objectives is to make the carbon footprint more visible in order to first raise awareness and then to launch actions for improvements to reduce the impact of data transmission on RENATER network.
The perimeter includes network devices such as routers, optical devices such as OTN switches and WDM multiplexers, optical fiber and network supervision services (NOC) which include the devices used for the supervision. Local networks and user devices are not considered.

We tested two conditions for data transmission, varying:

- Infrastructure density: We study a segment in a dense area (in Paris region) and a segment crossing France (Orsay Montpellier).

- Network activity: We assess the impacts of transmission on a peak day and an off-peak day. Thus, we perform direct measures on a peak day such as Tuesday or Thursday and on an off-peak day such as Sunday, because we perform measures on a network dedicated to the French Education and Research community.

After reviewing related work (Section II), we present the scope of our study (Section III). Then, we detail the environment developed to get direct measures on the network devices (Section IV), and the model chosen to evaluate the carbon footprint of a data transmission (Section V). Finally, we present and discuss the results obtained, and provide leads for future work (Section VII)

\section{RELATED WORK}

[1] performed a complete LCA of data transmission on an backbone network. They study a Swedish network and take into account: optical fiber cable production, deployment and use; routers, switches and other network link elements. The paper focuses mostly on energy consumption since the authors explain that it has the largest contribution by far in terms of Global Warming Potential (GWP). This LCA is done for the total IP core Swedish network, which does not allow to study the infrastructure and activity impacts. Their results show that, for Sweden, cable deployment has the highest impact, followed by equipment manufacturing and use stage, whereas with the world average electricity mix, use stage has by far the highest impact (Sweden has a very low carbon intensity for electricity).

Other studies are entirely focused on the electricity consumption implied by data transmission on the Internet, by 
assessing the electricity intensity of Internet, i.e., the electricity consumed per amount of data transmitted. [2] did a comparative study of 14 papers which estimated the average electricity intensity of transmitting data through fixed-line Internet. They show that differences in system boundary, assumptions used and the year considered by the study significantly affect the results. By using the data they considered most relevant and updating it, the authors propose a value for the electricity intensity of data transmission of $0.06 \mathrm{kWh} / \mathrm{GB}$ for 2015 , based on BT network in the UK. One of the papers referenced in the previous study, [3], used a methodology similar to ours, in so that it is also based on direct measures. Yet, it only measures the electricity intensity and is restricted to a specific application, a Internet video transmission.

\section{SCOPE: SYSTEM AND GHG EMISSIONS CONSIDERED}

\section{A. Scope}

We describe here the goal and scope of this simplified LCA.

The functional unit is the following: "Transmit $1 \mathrm{~GB}$ of data between Orsay and Montpellier (resp. Orsay and Jussieu) via a fiber optic link". Orsay and Jussieu are located in dense, geographically close areas in the Paris region. Montpellier is in the South of France, approximately $700 \mathrm{~km}$ from Paris.

Given the lack of reliable data on other impact indicators, only GHG emissions are considered, expressed in $\mathrm{kgs}$ of carbon dioxide equivalent $\left(\mathrm{CO}_{2} \mathrm{e}\right)$.

We have included emissions due to the manufacturing of equipment.

The system includes the transmission network only, i.e., from the sender institution's RENATER access point to the receiver institution's RENATER access point, including the network supervision devices. Fig 1 represents the system modeled between Orsay and Montpellier.

The system was broken down into 3 blocks, which have different characteristics in terms of emissions calculation, as we will see later:

- Core network except optical fiber, which is mainly composed of routers, OTN switches and WDM optical devices.

- Optical fiber.

- Network supervision devices, called NOC.

The studied system does not take into account the following elements:

- GHG emissions generated by the manufacturing of the buildings housing the equipment.

- GHG emissions generated by the end of life of the devices of the study, in particular recycling, due to the lack of reliable data.

- GHG emissions generated by the transportation of individuals involved in design, engineering and maintenance within RENATER and the NOC.

\section{B. GHG emissions}

GHG emissions generated by the transport of a gigabyte on the network result from:

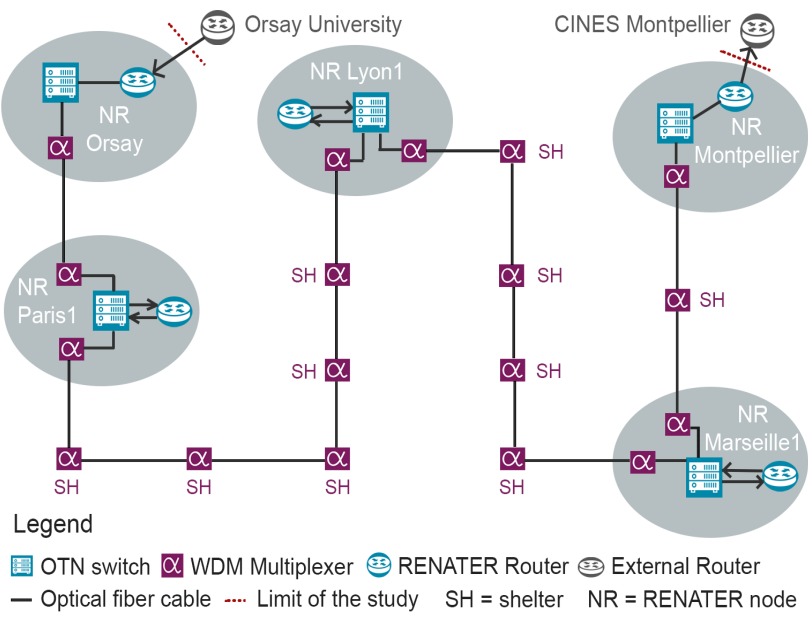

Fig. 1. Network model between Orsay and Montpellier

1) GHG emissions generated by the production (manufacturing, distribution, installation) of all core network and supervision devices. The NOC part is reduced to the share of $1 \mathrm{~GB}$ transmission on the total data traffic on RENATER network.

2) GHG emissions generated by the use of the same devices.

Estimating these emissions required two main pieces of information for each equipment: its energy consumption and its traffic over the same period. We set up the MESDIR environment to evaluate them on RENATER network, which is described in the next section.

\section{MESDIR: IMPACT ASSESSMENT ENVIRONMENT}

\section{A. Environment description}

To evaluate data transmission impact, we created a comprehensive environment for direct measures on the network and transmission devices of the RENATER backbone. We identified the typical route for each segment, and the RENATER devices involved, then performed direct measures on these devices. Tables I and II list the devices for each segment.

TABLE I

ORSAY-JUSSIEU SEGMENT DEVICES

\begin{tabular}{|c|c|c|}
\hline \multicolumn{3}{|c|}{ Orsay-Jussieu segment (3 hops) } \\
\hline Site & Equipment & OS / Model \\
\hline \multirow{3}{*}{ Orsay } & Router Cisco ASR 9910 & IOS-XR 6.6.3 \\
\cline { 2 - 3 } & WDM Multiplexer Infinera & mTera \\
\cline { 2 - 3 } & OTN Switch Infinera & Hit 7300 \\
\hline \multirow{3}{*}{ Paris1 1} & Router Juniper MX2010 & JunOS 18.4R1.8 \\
\cline { 2 - 3 } & WDM Multiplexer Infinera & mTera \\
\cline { 2 - 3 } & OTN Switch Infinera & Hit 7300 \\
\hline \multirow{3}{*}{ Jussieu } & Router Cisco ASR 9910 & IOS-XR 6.6.3 \\
\cline { 2 - 3 } & WDM Multiplexer Infinera & mTera \\
\cline { 2 - 3 } & OTN Switch Infinera & Hit 7300 \\
\hline
\end{tabular}


TABLE II

ORSAY-MONTPELLIER SEGMENT DEVICES

\begin{tabular}{|c|c|c|}
\hline \multicolumn{3}{|c|}{ Orsay-Montpellier segment (5 hops) } \\
\hline Site & Equipment & OS / Model \\
\hline \multirow{3}{*}{ Orsay } & Router Cisco ASR 9910 & IOS-XR 6.6.3 \\
\cline { 2 - 3 } & WDM Multiplexer Infinera & mTera \\
\cline { 2 - 3 } & OTN Switch Infinera & Hit 7300 \\
\hline \multirow{3}{*}{ Paris1 } & Router Juniper MX2010 & JunOS 18.4R1.8 \\
\cline { 2 - 3 } & WDM Multiplexer Infinera & mTera \\
\cline { 2 - 3 } & WDM Multiplexer Infinera & Hit 7300 \\
\cline { 2 - 3 } Lyon1 & OTN Switch Infinera & Hit 7300 \\
\hline \multirow{4}{*}{ Marseille1 } & Router Juniper MX2010 & JunOS 18.4R1.8 \\
\cline { 2 - 3 } & WDM Multiplexer Infinera & mTera \\
\cline { 2 - 3 } & WDM Multiplexer Infinera & Hit 7300 \\
\cline { 2 - 3 } & OTN Switch Infinera & Hit 7300 \\
\cline { 2 - 3 } & Router Juniper MX2010 & JunOS 18.4R1.8 \\
\cline { 2 - 3 } & WDM Multiplexer Infinera & mTera \\
\cline { 2 - 3 } & OTN Swittiplexer Infinera & Hit 7300 \\
\hline \multirow{3}{*}{ Montpellier } & Router Cisco ASR 9910 & Hit 7300 \\
\cline { 2 - 3 } & WDM Multiplexer Infinera & mR 6.6.3 \\
\cline { 2 - 3 } & OTN Switch Infinera & Hit 7300 \\
\hline
\end{tabular}

\section{B. Experiment description}

To obtain the IP traffic and the energy consumption of each equipment, we used Redhat Ansible ${ }^{1}$ to automatically get and gather the real data from the devices. To parse values and generate results, we used the templating language Jinja2 2 . We chose Ansible because it has an agentless approach suitable for the case of network devices and natively supports the Routers operating systems (OS): IOS, IOSXR and JunOS 3 . We also implemented a continuous integration mechanism using Gitlab CI/CD to automate the generation of results. ${ }^{4}$.

Data transmission on the RENATER backbone can be modeled and grouped into three blocks: the transmitter block, the forwarding block (including signal amplifications (shelters)) and the receiver block. For the IP traffic, we set up two measurements: one at midnight and one at $11: 50 \mathrm{pm}$. The collected data is the incoming and the outgoing data per interface and per router, then we calculated the difference between the 11:50 pm and midnight collection to obtain the number of bytes through the interface per day. We calculated the IP traffic for each equipment as follows:

- The IP traffic for a router $=$ the sum of the averages of incoming and outgoing data from the router's interfaces during the day.

- The IP traffic for an OTN switch on the site using one router $=$ the average of the IP traffic (incoming and outgoing) for the router on site during the day.

- The IP traffic for an OTN switch on the site using two routers $=$ sum of the averages of the IP traffic (incoming and outgoing) of both routers on site during the day.

- The IP traffic for a WDM Multiplexer = the sum of the averages of incoming and outgoing data of the router interfaces associated with the WDM links during the day.

\footnotetext{
${ }^{1}$ https://docs.ansible.com/ last accessed: July 2020.

${ }^{2}$ https://jinja.palletsprojects.com/en/2.11.x/ last accessed: July 2020.

${ }^{3}$ https://docs.ansible.com/ansible/latest/collections/ last accessed: July 2020.

${ }^{4}$ https://docs.gitlab.com/ce/ci/introduction/ last accessed : July 2020.
}

We did the direct measures in June 2020. During this month, the IP traffic was still impacted by the exceptional sanitary situation of the COVID-19, so the traffic figures were lower than usual on RENATER network. It was the reverse for the mass consumer Internet access providers.

The results of the IP traffic of the routers of the segment Orsay-Montpellier are illustrated in Fig 2.

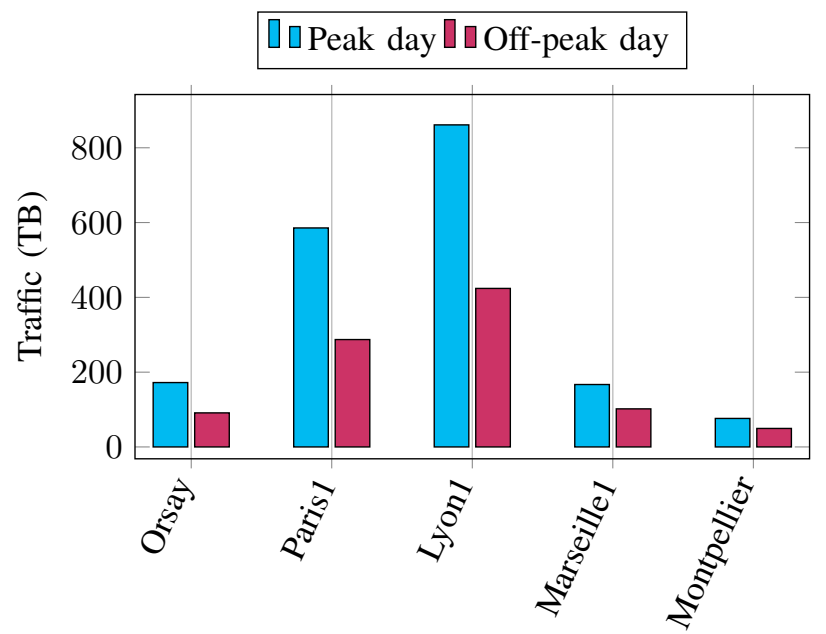

Fig. 2. Routers traffic measured on Orsay-Montpellier segment during one day

For the electricity consumption, we set up an hourly measurement during one day for all devices of our study. Results for the routers of the segment Orsay-Montpellier are illustrated in Fig 3.

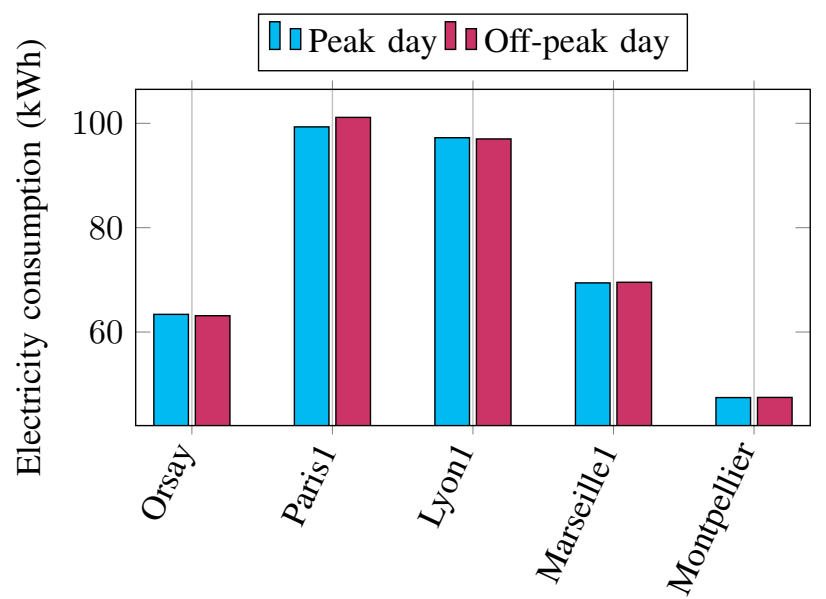

Fig. 3. Electricity consumption measured on Orsay-Montpellier segment during one day

\section{Results description}

To obtain the environmental impact of a GB, an intermediary step is to calculate the energy intensity by GB by equipment. For each equipment, we divide its electricity consumption by its IP traffic.

Values for the energy intensity in [2] range from 0.004 to $136 \mathrm{kWh} / \mathrm{GB}$. Our measures give an energy intensity of resp. 
0.007 and $0.002 \mathrm{kWh} / \mathrm{GB}$ for off-peak and peak days, which is in the low range for energy intensity.

Having direct measures on two typical days of the network activity (peak and off-peak day) enabled us to verify that the energy consumption is quite stable even though the traffic varies a lot, and increases with the number of hops.

\section{IMPACT ASSESSMENT}

\section{A. Model}

For most devices except the optical fiber, for which the calculations will be explained later, the calculation of GHG impacts has been separated into: production impact (including material extraction, manufacturing and distribution) and use stage impact. In addition, we calculated the carbon impacts of the NOC and the optical fiber. The general calculation of the carbon impact of $1 \mathrm{~GB}$ is the following equation.

impact $_{1 G B}=\sum_{e \in E}\left(I_{\text {production }}(e)+I_{\text {use }}(e)\right)+I_{N O C}+I_{\text {fiber }}$

with:

- $E$ the set of devices for the network part considered.

- $I_{\text {production }}(e)$ the GHG emissions due to the production of equipment $e$.

- $I_{\text {use }}(e)$ the GHG emissions due to the use of equipment $e$.

- $I_{\text {NOC }}$ the GHG emissions due to the NOC for $1 \mathrm{~GB}$.

- I $I_{\text {fiber }}$ the GHG emissions due to the fiber for one $1 \mathrm{~GB}$.

The following assumptions were made:

- The Power Usage Effectiveness (PUE) of the network sites is considered to be 1.8. [2] indicated that the average PUE was between 1.8 and 2.0. As energy efficiency improves over time, the PUE value we chose is the proposed low average PUE value of 1.8.

- The lifetime of the equipment corresponds to the duration of use on the RENATER network. This choice was made because there is no follow-up on the computer equipment once it has been delivered to a computer equipment sorting center. Routers lifetime value is 10 years, and optical devices lifetime value 12 years.

- When assessing the GWP part due to equipment production, the IP traffic transiting on an equipment is considered to be constant over the lifetime of the equipment. This assumption was made because traffic evolution is not known.

For a given network block, its production impact is considered to be the sum of the production impacts of each equipment divided by the total traffic on this equipment over its lifetime, as detailed in the following equation.

$$
I_{\text {production }}=\sum_{e \in E} \frac{I_{\text {production }}(e)}{T(e, 1 \text { year }) \times L(e)}
$$

with:

- $E$ the set of devices for the network part considered.

- $I_{\text {production }}(e)$ the GHG emissions due to the production of equipment $e$.
- $L(e)$ the lifetime in years of equipment $e$.

- $T(e, 1$ year $)$ the traffic on equipment $e$ in one year.

GHG emissions due to the production of network devices are either taken from manufacturer data or extrapolated from manufacturer data. For the NOC devices, we used the Ecodiag ${ }^{5}$ tool.

Traffic data comes from RENATER (MESDIR environment).

Use stage impact is based on the energy consumption of each equipment for a given duration (1 year for the NOC, 1 day for the backbone network) and the traffic on this equipment on the same duration. The energy consumption of the backbone devices is the average of the direct measures of the studied day.

$$
I_{u s e}=\sum_{e \in E} \frac{C(e, d) \times P U E(e)}{T(e, d)} \times E F
$$

with:

$-d$ the duration considered (1 day or 1 year)

- $C(e, d)$ the energy consumption of equipment $e$ for duration $d$, in $\mathrm{kWh}$.

- $T(e, d)$ the traffic on equipment $e$ during duration $d$, or supervised by equipment $e$ in the case of NOC devices.

- PUE the PUE of the equipment datacenter when relevant, which was here actually considered a constant, as specified in III, and 1 otherwise.

- EF the emission factor of electricity for France, in $\mathrm{kg} \mathrm{CO}_{2} \mathrm{e} / \mathrm{kWh}$. We use $\mathrm{EF}=0.108 \mathrm{kgCO} 2 \mathrm{e} / \mathrm{kWh}$ from ELDC database.

Electricity consumption mostly comes from direct measures from RENATER (MESDIR environment), except from the devices in shelters, for which we could not get direct measures. For these devices, we estimated the consumption based on the maximum power given in the documentation. Lifetimes of devices and annual traffic were also provided by RENATER.

The impact of the optical fiber is described on the following equation.

$$
I_{\text {fiber }}=\frac{E F_{\text {fiber }} \times \# k m}{L_{\text {fiber }} \times T(\text { total network, } 1 \text { year })}
$$

with :

- $E F_{\text {fiber }}$ the emission factor of the optical fiber in $\mathrm{kgCO} 2 \mathrm{e} / \mathrm{km}$. We use $E F_{\text {fiber }}=1.27 \mathrm{kgCO} 2 \mathrm{e} / \mathrm{m}$ from ACOME manufacturer for a cable with 96 fibers.

- $L_{\text {fiber }}$ the lifetime of the optical fiber in years.

- \#km the length of optical fiber on the network.

- T(total network, 1 year) the total traffic of the network during one year.

\section{RESUlts}

The optical fiber and NOC impacts measures are the average of the impact of $1 \mathrm{~GB}$ under the whole traffic of RENATER network. The results are the following :

- The optical fiber impact is $2.59 \mathrm{E}-05 \mathrm{kgCO} 2 \mathrm{e} / \mathrm{GB}$.

- The NOC impact is $3.73 \mathrm{E}-05 \mathrm{kgCO} 2 \mathrm{e} / \mathrm{GB}$.

\footnotetext{
${ }^{5}$ https://ecoinfo.cnrs.fr/ecodiag/ last accessed: May 2020.
} 
Concerning the core network, its impacts are given in Table III. The contributions of each part are summed up in Fig 4.

TABLE III

CORE NETWORK IMPACTS IN $\mathrm{KGCO}_{2}$ E/GB

\begin{tabular}{|c|c|c|c|}
\hline Segment & Activity & Use & Production \\
\hline Orsay-Montpellier & peak day & $7.93 \mathrm{E}-04$ & $4.93 \mathrm{E}-04$ \\
\hline & off-peak day & $1.40 \mathrm{E}-03$ & $4.93 \mathrm{E}-04$ \\
\hline Orsay-Jussieu & peak day & $4.33 \mathrm{E}-04$ & $1.43 \mathrm{E}-04$ \\
\hline & off-peak day & $8.45 \mathrm{E}-04$ & $1.43 \mathrm{E}-04$ \\
\hline
\end{tabular}

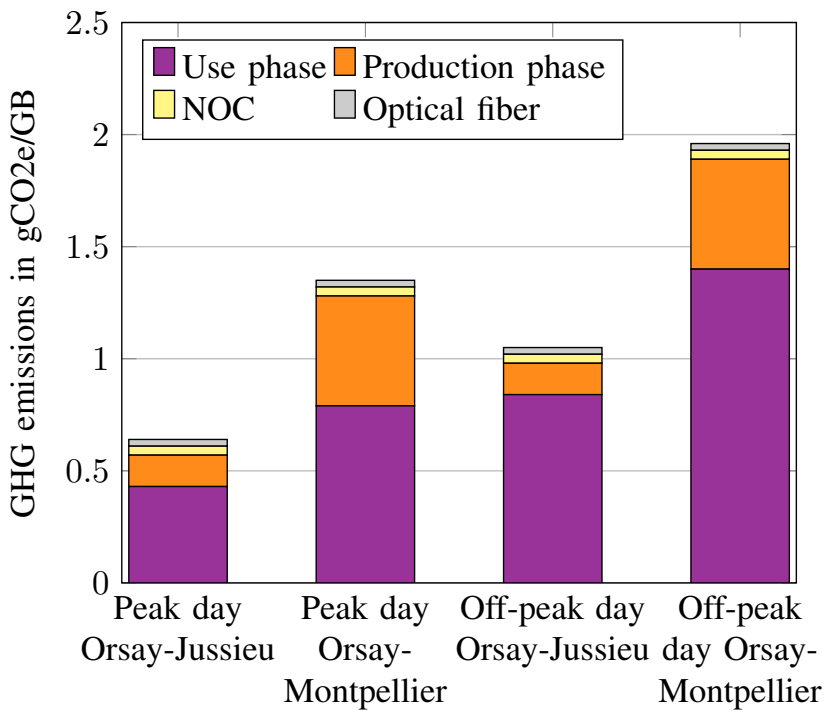

Fig. 4. Impact contributions in terms of GHG emissions percentage

Like [4], we found that the impact of the use phase is the highest for each case scenario (above 59\%), followed by the production phase. The impact of the NOC is just above the impact of the optical fiber in each scenario.

During an off-peak day, the impact of data transmission is higher than during a peak day, correlated to the energy intensity of a GB. Therefore, one challenge will be to focus on scheduling flows over time and sharing bandwidth.

The impact of data transmission is higher for the segment with more hops and a lower density.

Sensitivity analyses also show that the lifetime of the devices has a strong influence. Increasing the lifetime of the devices reduces the production impact. Therefore, it reduces the carbon footprint of the transmission.

The emission factor of electricity has a strong impact on the final results. This factor varies a lot with the geographical location, which makes it essential to choose a low factor source.

The traffic transiting on the devices has a significant impact. All the devices of the network are built to handle a larger quantity of data, generally 50 times more. It is essential to maximize the traffic on them.

One major limitation of this study is the lack of inclusion of the end-of-life phase due to lack of reliable data.

\section{CONCLUSION AND PERSPECTIVES}

In this paper, we have estimated the GHG emissions associated with the transfer of $1 \mathrm{~GB}$ of data on the RENATER backbone, based on a simplified LCA methodology.

We implemented an environment to get direct measures through RENATER backbone. This environment automatically measures the energy consumption and traffic of the backbone devices required for the data transmission. We tested the impacts on the transmission of two conditions: the infrastructure density of the network (considering two segments to get direct measures the first in the dense area of Paris and the second crossing France) and the network activity (performing measures a peak day and on an off-peak day). Then, we used these direct measures as inputs for our model to assess the GHG emissions of the transmission of $1 \mathrm{~GB}$. The estimated impacts take into consideration the impacts of the production and the use phases of all the devices of the study. Our results verify that both conditions have a carbon impact on the transmission of $1 \mathrm{~GB}$.

This study allowed RENATER to better understand the environmental impacts related to its backbone network. Several major vectors are possible and could be taken into account in the next architectures of RENATER backbone. The results provide keys for RENATER to assess the carbon impact of all online services, such as e-mail and videoconferencing. In addition, our work is also a basis for building a real-time carbon monitoring tool for the backbone network segments.

To complete our study, it would be interesting to take into account other parameters such as the temperature of the equipment and the temperature of the server room. On the other hand, more time and resources are needed to add other environmental indicators, such as the amount of water used, the amount of resources used, the impact on pollution (air, water and soil), etc., which are poorly documented.

\section{ACKNOWLEDGMENTS}

We thank RENATER for supplying all the resources and data for the study, and the GDS EcoInfo for their expertise. Several suppliers also provided us data necessary for our research.

\section{REFERENCES}

[1] J. Malmodin, D. Lundén, M. Nilsson, and G. Andersson, "LCA of data transmission and IP core networks," in 2012 Electronics Goes Green $2012+$. IEEE, 2012, pp. 1-6.

[2] J. Aslan, K. Mayers, J. G. Koomey, and C. France, "Electricity intensity of internet data transmission: Untangling the estimates," Journal of Industrial Ecology, vol. 22, no. 4, pp. 785-798, 2018.

[3] V. C. Coroama, L. M. Hilty, E. Heiri, and F. M. Horn, "The direct energy demand of internet data flows," Journal of Industrial Ecology, vol. 17, no. 5, pp. 680-688, 2013.

[4] K. Grobe, "Environmental challenges on photonics networks equipment," in Photonic Networks; 18. ITG-Symposium. VDE, 2017, pp. 1-5. 